\title{
Multiplicative-accumulative matching of NLO calculations with parton showers
}

\author{
Paolo Nason ${ }^{a, b}$ and Gavin P. Salam ${ }^{c, d}$ \\ ${ }^{a}$ Università di Milano-Bicocca and INFN, Sezione di Milano-Bicocca, \\ Piazza della Scienza 3, 20126 Milano, Italy \\ ${ }^{b}$ INFN, Sezione di Milano-Bicocca, \\ Piazza della Scienza 3, 20126 Milano, Italy \\ ${ }^{c}$ Rudolf Peierls Centre for Theoretical Physics, University of Oxford, Clarendon Laboratory, \\ Parks Road, Oxford OX1 3PU, United Kingdom \\ ${ }^{d}$ All Souls College, \\ Oxford $O X 14 A L$, United Kingdom \\ E-mail: paolo.nason@mib.infn.it, gavin.salam@physics.ox.ac.uk
}

ABSTRACT: We propose a new approach for combining next-to-leading order (NLO) and parton shower (PS) calculations so as to obtain three core features: (a) applicability to general showers, as with the MC@NLO and POWHEG methods; (b) positive-weight events, as with the KrkNLO and POWHEG methods; and (c) all showering attributed to the parton shower code, as with the MC@NLO and KrkNLO methods. This is achieved by using multiplicative matching in phase space regions where the shower overestimates the matrix element and accumulative (additive) matching in regions where the shower underestimates the matrix element, an approach that can be viewed as a combination of the MC@NLO and KrkNLO methods.

Keywords: NLO Computations, QCD Phenomenology

ArXiv EPrint: 2111.03553 


\section{Contents}

1 Introduction 1

2 POWHEG, MC@NLO and KrkNLO 2

3 The new method 5

4 Variants of the method $\quad 6$

$\begin{array}{llr}5 & \text { Conclusion } & 8\end{array}$

\section{Introduction}

Next-to-leading (NLO) order event generators interfaced to Parton Showers (NLOPS from now on) have, in the past decades, become the state of the art for the simulation of hard collider processes. The MC@NLO algorithm was the first one to be proposed [1]. Soon after, the POWHEG method was proposed [2] in order to overcome the problem of negative weights inherent to MC@NLO. Novel methods were proposed later [3-5]. Among them, the KrkNLO method, like the POWHEG method, has the characteristic of being free from negative weights, although its applicability is at the moment restricted to relatively simple processes.

Both the MC@NLO and the KrkNLO methods are intimately intertwined with the parton shower generator that is adopted. In fact, the two methods provide the corrections one must apply to the parton shower result in order to achieve next-to-leading order (NLO) accuracy. This is at variance with POWHEG, which is largely independent from the shower generator. In fact, POWHEG takes care of the generation of the hardest event in such a way that NLO accuracy is preserved, while the PS takes care of the remaining (less hard) radiation. This feature is convenient, since one can generate events in the Les Houches format [6] and then shower them with any available PS generator that complies with the Les Houches format specifications.

In view of recent developments aimed at the improvement of the logarithmic accuracy of the shower (see refs. [7-10], [11-13] and $[14,15]$ ) it is legitimate to ask whether POWHEG will maintain the same advantage, i.e. whether minor modifications to the POWHEG algorithm will be sufficient to guarantee that, when interfaced to an already NLL accurate shower, NLL accuracy will be maintained, or whether it will be more feasible to correct an already NLL accurate shower to achieve also NLO accuracy, or rather whether both alternatives will be feasible, and will productively compete among each other. It is therefore important to explore the full range of options for matching NLO calculations and parton showers.

In this note, we will address certain limitations of MC@NLO and KrkNLO, and show how they can be overcome. The MC@NLO approach is widely applicable, and widely used, but 
has the undesirable feature of negative weights. ${ }^{1}$ On the other hand, the KrkNLO method has positive weights, but it is difficult to extend it to generic processes. We will show that combining the two methods one could achieve both positive weights and unrestricted applicability.

The paper is organised as follows, in section 2 we will compare the POWHEG, MC@NLO and KrkNLO methods by formulating them in a common language. We will do this by extending the formulation of the POWHEG and MC@NLO methods of ref. [19] also to the KrkNLO method. In section 3 we will present the combined method, and in section 4 we will present some of its possible variants. Finally, in section 5 we present our conclusions.

\section{POWHEG, MC@NLO and KrkNLO}

We assume that the phase space with radiation $\Phi$ can be written in terms of an underlying Born phase space $\Phi_{\mathrm{B}}$ and three radiation variables, indicated collectively as $\Phi_{\text {rad }}$. We also assume that the mapping from $\Phi$ to $\Phi_{\mathrm{B}}$ is such that in the singular (collinear or soft) limit, the Born configuration matches the full phase space with the collinear pair merged into a single parton or with the soft particle removed. Such mappings are easy to realise for processes with a single singular region, while for more complex processes one must separate the real cross section into contributions having a single singular region. In the following illustrative discussion we ignore these complications.

We define the following quantity, a function of the underlying Born kinematics,

$$
\bar{B}_{\mathrm{S}}\left(\Phi_{\mathrm{B}}\right)=B_{0}\left(\Phi_{\mathrm{B}}\right)+V\left(\Phi_{\mathrm{B}}\right)+\int R_{\mathrm{S}}(\Phi) \mathrm{d} \Phi_{\mathrm{rad}}
$$

where the full phase space $\Phi$ is defined as function of $\Phi_{\mathrm{B}}$ and $\Phi_{\mathrm{rad}}, B_{0}$ is the Born cross section, $V$ comprises the virtual corrections and, for hadron initiated processes, the collinear counterterms integrated at fixed underlying Born, and $R_{\mathrm{S}}$ is a part of the real cross section that includes all soft and collinear singularities. In other words, $R-R_{\mathrm{S}}$ is non-singular. Notice that if $R_{\mathrm{S}}$ is taken equal to $R, \bar{B}_{\mathrm{S}}$ is the inclusive cross section at fixed underlying Born. We imagine that renormalisation has been carried out, and that the infrared divergences arising in $V$ and in the $\mathrm{d} \Phi_{\text {rad }}$ integral of $R_{s}$ are regularised in some way. Notice also that $\bar{B}_{\mathrm{S}}$ is finite, since the singularities present in $V$ cancel those arising integrating the $R_{\mathrm{S}}$ term.

We also define the Sudakov form factor associated with $R_{\mathrm{S}}$

$$
S\left(t, \Phi_{\mathrm{B}}\right)=\exp \left[-\int_{t_{\Phi}>t} \frac{R_{\mathrm{S}}(\Phi)}{B_{0}\left(\Phi_{\mathrm{B}}\right)} \mathrm{d} \Phi_{\mathrm{rad}}\right]
$$

where $\Phi$ is defined in terms of the variables $\Phi_{\mathrm{B}}$ and $\Phi_{\mathrm{rad}}$, and $t$ is some definition of hardness, depending upon the full phase space with radiation. One may think, for example, that $t$ is the relative transverse momentum of the splitting pair. If $R_{\mathrm{S}}$ was taken equal to $R$, the Sudakov form factor would represent the probability for not radiating anything harder than $t$.

\footnotetext{
${ }^{1}$ Recent proposals for the reduction of the negative-weight fraction include refs. [16-18].
} 
The hardest event cross section can be represented in both POWHEG and MC@NLO as

$$
\begin{aligned}
\mathrm{d} \sigma= & \bar{B}_{\mathrm{S}}\left(\Phi_{\mathrm{B}}\right) S\left(t_{\text {cut }}, \Phi_{\mathrm{B}}\right) \mathrm{d} \Phi_{\mathrm{B}}+\bar{B}_{\mathrm{S}}\left(\Phi_{\mathrm{B}}\right) S\left(t_{\Phi}, \Phi_{\mathrm{B}}\right) \times \frac{R_{\mathrm{S}}(\Phi)}{B_{0}\left(\Phi_{\mathrm{B}}\right)} \theta\left(t_{\Phi}-t_{\text {cut }}\right) \mathrm{d} \Phi+ \\
& +\left[R(\Phi)-R_{\mathrm{S}}(\Phi)\right] \mathrm{d} \Phi,
\end{aligned}
$$

where $\mathrm{d} \Phi=\mathrm{d} \Phi_{\mathrm{B}} \mathrm{d} \Phi_{\text {rad }}$, and $t_{\text {cut }}$ represents a lower limit for radiation, needed to avoid the Landau-pole singularities. Events are generated with a probability proportional to each term of the cross section formula. The first two terms are generated with a Monte Carlo technique. In fact, they satisfy the shower unitarity equation

$$
S\left(t_{\text {cut }}, \Phi_{\mathrm{B}}\right)+\int_{t_{\Phi}>t_{\text {cut }}} S\left(t_{\Phi}, \Phi_{\mathrm{B}}\right) \times \frac{R_{\mathrm{S}}(\Phi)}{B_{0}\left(\Phi_{\mathrm{B}}\right)} \mathrm{d} \Phi_{\mathrm{rad}}=1
$$

which follows from the fact that the expression under the integral sign is an exact differential. In POWHEG, the generation of the hardness $t$ is uniform in the Sudakov form factor, and one can generate events with the standard shower algorithm by equating a random number with the Sudakov form factor, and solving for $t$. If the $t$ value so obtained is above $t_{\text {cut }}$, the radiation kinematics is generated, and the event with the hardest radiation is fed to a shower generator, which takes care of adding subsequent (less hard) radiation.

In the case of MC@NLO, the implementation of the first two terms is generally more involved. If the shower is ordered in transverse momentum, the hardest emission is the first, and the radiation mechanism is the same as in POWHEG, except that it is implemented within the shower generator, rather than in the NLO program. In case of an angular ordered shower, large angle soft radiation is generated first, and the hardest radiation occurs somewhere down the shower. ${ }^{2}$ It was shown in ref. [2] that, in this case, by suitable rearrangement of the Sudakov factors for each emission, one reconstructs the transverse momentum Sudakov form factor in formula (2.3).

The last term in eq. (2.3) is non-singular, and thus is dominated by hard radiation. It is handled essentially in the same way in MC@NLO and POWHEG.

The NLO accuracy of eq. (2.3) can be demonstrated by computing the expectation value of a generic infrared safe observable $O(\Phi)$ as follows

$$
\begin{aligned}
\langle O\rangle=\int \mathrm{d} \Phi_{\mathrm{B}} \bar{B}_{\mathrm{S}}\left(\Phi_{\mathrm{B}}\right)\{ & \left.S\left(t_{\text {cut }}, \Phi_{\mathrm{B}}\right) O\left(\Phi_{\mathrm{B}}\right)+\int_{t_{\Phi}>t_{\text {cut }}} S\left(t_{\Phi}, \Phi_{\mathrm{B}}\right) \times \frac{R_{\mathrm{S}}(\Phi)}{B_{0}\left(\Phi_{\mathrm{B}}\right)} O(\Phi) \mathrm{d} \Phi_{\mathrm{rad}}\right\}+ \\
& +\int\left[R(\Phi)-R_{\mathrm{S}}(\Phi)\right] O(\Phi) \mathrm{d} \Phi
\end{aligned}
$$

or equivalently,

$$
\begin{gathered}
\langle O\rangle=\int \mathrm{d} \Phi_{\mathrm{B}} \bar{B}_{\mathrm{S}}\left(\Phi_{\mathrm{B}}\right)\left\{S\left(t_{\mathrm{cut}}, \Phi_{\mathrm{B}}\right) O\left(\Phi_{\mathrm{B}}\right)+\int_{t_{\Phi}>t_{\mathrm{cut}}} S\left(t_{\Phi}, \Phi_{\mathrm{B}}\right) \times \frac{R_{\mathrm{S}}(\Phi)}{B_{0}\left(\Phi_{\mathrm{B}}\right)} O\left(\Phi_{\mathrm{B}}\right) \mathrm{d} \Phi_{\mathrm{rad}}+\right. \\
\left.\quad+\int_{t_{\Phi}>t_{\mathrm{cut}}} S\left(t_{\Phi}, \Phi_{\mathrm{B}}\right) \times \frac{R_{\mathrm{S}}(\Phi)}{B_{0}\left(\Phi_{\mathrm{B}}\right)}\left[O(\Phi)-O\left(\Phi_{\mathrm{B}}\right)\right] \mathrm{d} \Phi_{\mathrm{rad}}\right\}+ \\
+\int\left[R(\Phi)-R_{\mathrm{S}}(\Phi)\right] O(\Phi) \mathrm{d} \Phi
\end{gathered}
$$

\footnotetext{
${ }^{2}$ Taking $t$ to be the angular scale of the shower means that $t$ does not represent a hardness. As a result, in the absence of an infrared cutoff, the first emission is dominated by the infinitely soft region (where $R_{\mathrm{S}}=R$ ) rather than by the hard region.
} 
In view of the unitarity equation (2.4), the first two terms in the curly bracket of equation (2.5b) collapse into $O\left(\Phi_{\mathrm{B}}\right)$. Furthermore, the factor $\left[O(\Phi)-O\left(\Phi_{\mathrm{B}}\right)\right]$ in the third term in the curly bracket suppresses the singular region, so that up to higher order terms the Sudakov form factor can be omitted, and the lower integration limit can be set to zero up to a power-suppressed correction. We thus get

$$
\begin{aligned}
\langle O\rangle=\int \mathrm{d} \Phi_{\mathrm{B}} \bar{B}_{\mathrm{S}}\left(\Phi_{\mathrm{B}}\right)\left\{O\left(\Phi_{\mathrm{B}}\right)+\right. & \left.\int \frac{R_{\mathrm{S}}(\Phi)}{B_{0}\left(\Phi_{\mathrm{B}}\right)}\left[O(\Phi)-O\left(\Phi_{\mathrm{B}}\right)\right] \mathrm{d} \Phi_{\mathrm{rad}}\right\}+ \\
& +\int\left[R(\Phi)-R_{\mathrm{S}}(\Phi)\right] O(\Phi) \mathrm{d} \Phi+\mathcal{O}(\mathrm{NNLO}) \\
=\int \mathrm{d} \Phi_{\mathrm{B}}\left\{\bar{B}_{\mathrm{S}}\left(\Phi_{\mathrm{B}}\right) O\left(\Phi_{\mathrm{B}}\right)+\right. & \left.\int R_{\mathrm{S}}(\Phi)\left[O(\Phi)-O\left(\Phi_{\mathrm{B}}\right)\right] \mathrm{d} \Phi_{\mathrm{rad}}\right\}+ \\
& +\int\left[R(\Phi)-R_{\mathrm{S}}(\Phi)\right] O(\Phi) \mathrm{d} \Phi+\mathcal{O}(\mathrm{NNLO}) .
\end{aligned}
$$

Inserting into the above equation the expression for $\bar{B}_{\mathrm{S}}$, eq. (2.1), we get

$$
\langle O\rangle=\int \mathrm{d} \Phi_{\mathrm{B}}\left[B\left(\Phi_{\mathrm{B}}\right)+V\left(\Phi_{\mathrm{B}}\right)\right] O\left(\Phi_{\mathrm{B}}\right)+\int R(\Phi) O(\Phi) \mathrm{d} \Phi+\mathcal{O}(\mathrm{NNLO}),
$$

which is the correct NLO expression for the observable.

POWHEG implements eq. (2.3) directly. $R_{\mathrm{S}}$ is defined as

$$
R_{\mathrm{S}}(\Phi)=F(\Phi) R
$$

where $F(\Phi) \leq 1$, and approaches 1 as $\Phi$ approaches the singular region, so that $R_{\mathrm{S}}$ carries all the singularity structure of $R$.

In MC@NLO, $R_{\mathrm{S}}$ is the shower approximation to $R$, typically given by the Born term times a DGLAP splitting function. Thus, in MC@NLO one computes a Born configuration using the $\bar{B}_{\mathrm{S}}$ function, and passes it directly to the Shower Monte Carlo. The $R-R_{\mathrm{S}}$ terms are generated separately, and fed directly to the shower. Negative weights can arise at this stage, since there is no guarantee that $R-R_{\mathrm{S}}$ is positive.

Using the same language adopted here for POWHEG and MC@NLO, the key formula for the KrkNLO method can be written as follows

$$
\mathrm{d} \sigma=\bar{B}_{\mathrm{S}}\left(\Phi_{\mathrm{B}}\right) S\left(t_{\text {cut }}, \Phi_{\mathrm{B}}\right) \mathrm{d} \Phi_{\mathrm{B}}+\bar{B}_{\mathrm{S}}\left(\Phi_{\mathrm{B}}\right)\left\{S\left(t_{\Phi}, \Phi_{\mathrm{B}}\right) \times \frac{R_{\mathrm{S}}(\Phi)}{B_{0}\left(\Phi_{\mathrm{B}}\right)}\right\} \times\left[\frac{R(\Phi)}{R_{\mathrm{S}}(\Phi)}\right] \mathrm{d} \Phi,
$$

(with an implicit $\theta\left(t-t_{\text {cut }}\right)$ in $\left.d \Phi\right)$. In the literature dealing with the KrkNLO method [5, 2025], particular emphasis has been put on the use of a specific scheme for the parton densities, which considerably simplifies the expression for the $\bar{B}_{\mathrm{S}}$ function. Here we are instead interested in a simpler aspect of the method, which is that to generate NLO accurate radiation, it uses a multiplicative correction, rather than the additive correction of the MC@NLO method. The NLO accuracy of the KrkNLO formula can be simply demonstrated by showing that KrkNLO is equivalent to MC@NLO at the NLO level. In fact, we can rewrite 
formula (2.9) as

$$
\begin{aligned}
\mathrm{d} \sigma= & \bar{B}_{\mathrm{S}}\left(\Phi_{\mathrm{B}}\right) S\left(t_{\text {cut }}, \Phi_{\mathrm{B}}\right) \mathrm{d} \Phi_{\mathrm{B}}+\bar{B}_{\mathrm{S}}\left(\Phi_{\mathrm{B}}\right) S\left(t_{\Phi}, \Phi_{\mathrm{B}}\right) \times \frac{R_{\mathrm{S}}(\Phi)}{B_{0}\left(\Phi_{\mathrm{B}}\right)} \times\left[\frac{R(\Phi)}{R_{\mathrm{S}}(\Phi)}-1\right] \mathrm{d} \Phi+ \\
& +\bar{B}_{\mathrm{S}}\left(\Phi_{\mathrm{B}}\right) S\left(t_{\Phi}, \Phi_{\mathrm{B}}\right) \frac{R_{\mathrm{S}}(\Phi)}{B_{0}\left(\Phi_{\mathrm{B}}\right)} \mathrm{d} \Phi .
\end{aligned}
$$

The middle term is now insensitive to the soft region, because the factor in square brackets vanishes there, so we can drop the Sudakov form factor and, neglecting terms of NNLO size, set $\bar{B}_{\mathrm{S}} / B_{0}=1$. By doing this we recover exactly eq. (2.3).

The KrkNLO method leads to positive weighted events. On the other hand, unlike MC@NLO and POWHEG, its cross section at fixed underlying Born does not exactly match the corresponding fixed order result, but differs from it by NNLO terms. ${ }^{3}$ The KrkNLO method, however, generates weighted events, so, the unweighting efficiency may constitute a problem if one wants to apply the method to generic processes without having to do process-by-process adjustments. As a related problem, the shower generator may not cover the full radiation phase space. This is the same as saying that $R_{\mathrm{S}}$ can become zero in certain regions, in which case the method is not applicable. For these reasons, it seems difficult to apply the method to generic processes in automated framework, something that has been available in POWHEG and MC@NLO since a long time.

\section{The new method}

By comparing the MC@NLO and KrkNLO method using a common language, it becomes clear that the two methods have much in common, and can in fact be merged in such a way that the KrkNLO positivity is maintained, unweighted events can be generated on the fly, and no issues arise from the limited coverage of the phase space by the parton shower code. The merged method is defined by the following formula

$\mathrm{d} \sigma=\bar{B}_{\mathrm{S}}\left(\Phi_{\mathrm{B}}\right) S\left(t_{\Phi}, \Phi_{\mathrm{B}}\right) \times \frac{R_{\mathrm{S}}(\Phi)}{B_{0}\left(\Phi_{\mathrm{B}}\right)} \times\left\{1+\frac{R-R_{\mathrm{S}}}{R_{\mathrm{S}}} \theta\left(R_{\mathrm{S}}-R\right)\right\} \mathrm{d} \Phi+\theta\left(R-R_{\mathrm{S}}\right)\left[R-R_{\mathrm{S}}\right] \mathrm{d} \Phi$,

where from now, for compactness, we leave out the no-radiation term from our expressions (for completeness, they are reintroduced at the end of section 4). It is easy to see by inspection that this formula has the same NLO accuracy as MC@NLO. In fact, the $\theta$ function in the first term of eq. (3.1) is regular, and as such the Sudakov form factor multiplying it and the $\bar{B}_{\mathrm{S}} / B_{0}$ ratio can both be set to 1 up to NNLO corrections. Having done this, the contribution proportional to the $\theta$ function in the first term becomes identical to the last term, except for the theta function, which has the opposite sign in the argument. The two terms thus combine, and the theta function disappears, yielding the MC@NLO formula.

The method of eq. (3.1) is immediately seen to have positive weights, since the second term in eq. (3.1) is forced to be positive by the theta function, while the factor in the curly

\footnotetext{
${ }^{3}$ Although some authors consider this to be an undesirable feature, it does not constitute a real problem, since choices for uncontrolled NNLO terms are made, for example, when choosing the scales, and there is no preferred way to define an NLO result as far as the neglected NNLO terms are concerned.
} 
bracket of the first term is forced by the theta function to be less than 1 . Notice that regions of phase space not populated by the Monte Carlo only contribute to the additive term in the square bracket, since they have $R_{\mathrm{S}}=0$.

Let us consider how the method of eq. (3.1) might be implemented in a scenario where it is the parton shower that is responsible for generating $R_{\mathrm{S}}{ }^{4}$ We first consider the case of a parton shower ordered in a genuine hardness variable (for example, transverse momentum). As in the MCONLO approach, the NLO program is responsible for generating a sample of Born events, with a distribution corresponding to the correct $\bar{B}_{\mathrm{S}}\left(\Phi_{\mathrm{B}}\right) \mathrm{d} \Phi_{\mathrm{B}}$ weight. The event is communicated to the parton shower, which generates a first emission, i.e. accounting for a remaining factor $S\left(t_{\Phi}, \Phi_{\mathrm{B}}\right) \frac{R_{\mathrm{S}}(\Phi)}{B_{0}\left(\Phi_{\mathrm{B}}\right)} \mathrm{d} \Phi / \mathrm{d} \Phi_{\mathrm{B}}$ in eq. (3.1). Next, the parton shower communicates the event, with its first emission, back to the NLO program, which evaluates the contents of the curly bracket in eq. (3.1). The result of the evaluation is to be used as the weight of the generated event. Since it is bounded between zero and one, it can be easily used as an acceptance probability for the whole event, so that, in contrast to the case of the pure KrKNLO method, unweighted events can be generated on the fly. If the event is accepted, the parton shower then continues from that point, generating the remaining emissions. In addition, the NLO program is responsible for generating a second (positive definite) sample of events, corresponding to the $\theta\left(R-R_{\mathrm{S}}\right)$ term of eq. (3.1), covering the part of the real phase space where the shower $R_{\mathrm{S}}$ underestimates the true real matrix element. These events are passed to the parton shower code for normal showering.

The above scheme requires a more connected workflow between the parton shower and the NLO code than either of the MC@NLO or POWHEG approaches. Recall that those approaches pass a Les Houches Event file to the shower code, and then let the shower take over from there. In contrast, the scheme outlined above requires additional action from the NLO code after the first parton shower emission. However, this is simply a technical consideration, which in our view is a small price to pay for an approach that, like the MC@NLO scheme, leaves responsibility for the first emission with the parton shower, while eliminating negative weights.

A further remark concerns angular-ordered parton showers. For such showers, the application of the acceptance probability (factor in curly brackets in eq. (3.1)) after the first emission would not be correct. Instead, one might envisage an approach in which one runs the complete shower, uses a jet algorithm to map the full event to the real phase space and then applies the acceptance probability.

\section{Variants of the method}

Eq. (3.1) can be viewed as a special case of a family of approaches, parameterised by a constant $c \geq 1$,

$$
\begin{aligned}
\mathrm{d} \sigma= & \bar{B}_{\mathrm{S}}\left(\Phi_{\mathrm{B}}\right) S\left(t_{\Phi}, \Phi_{\mathrm{B}}\right) \times \frac{c R_{\mathrm{S}}(\Phi)}{B_{0}\left(\Phi_{\mathrm{B}}\right)} \times\left\{1+\frac{R-c R_{\mathrm{S}}}{c R_{\mathrm{S}}} \theta\left(c R_{\mathrm{S}}-R\right)\right\} \mathrm{d} \Phi+ \\
& +\theta\left(R-c R_{\mathrm{S}}\right)\left[R-c R_{\mathrm{S}}\right] \mathrm{d} \Phi .
\end{aligned}
$$

\footnotetext{
${ }^{4}$ Alternatively, one might also implement the approach in a POWHEG-style scheme, where the NLO program takes responsibility for the first emission, and $R_{\mathrm{S}}$ is not required to be smaller than $R$.
} 
This formula was obtained by replacing $R_{\mathrm{S}} \rightarrow c R_{\mathrm{S}}$ in all occurrences where it appears in eq. (3.1), except for the Sudakov form factor. First of all, we should convince ourselves that this formula has the correct behaviour near the soft limit, and that it is NLO accurate. This is seen immediately by writing (4.1) as

$$
\begin{aligned}
\mathrm{d} \sigma= & \bar{B}_{\mathrm{S}}\left(\Phi_{\mathrm{B}}\right) S\left(t_{\Phi}, \Phi_{\mathrm{B}}\right) \times \frac{c R_{\mathrm{S}}(\Phi)}{B_{0}\left(\Phi_{\mathrm{B}}\right)} \times\left\{1+\frac{R-c R_{\mathrm{S}}}{c R_{\mathrm{S}}}\right\} \mathrm{d} \Phi \\
& +\theta\left(R-c R_{\mathrm{S}}\right)\left(R-c R_{\mathrm{S}}\right)\left[1-\frac{\bar{B}_{\mathrm{S}}\left(\Phi_{\mathrm{B}}\right)}{B_{0}\left(\Phi_{\mathrm{B}}\right)} S\left(t_{\Phi}, \Phi_{\mathrm{B}}\right)\right] \mathrm{d} \Phi .
\end{aligned}
$$

The first line is equal to the KrkNLO formula, eq. (2.9) (leaving aside the $t_{\text {cut }}$, for simplicity). In the second line, the factor in square brackets is of order $\alpha_{S}$ and it multiplies a term dominated by the hard region (and thus also of order $\alpha_{S}$ ), since

$$
\theta\left(R-c R_{\mathrm{S}}\right)\left(R-c R_{\mathrm{S}}\right) \leq \theta\left(R-R_{\mathrm{S}}\right)\left(R-R_{\mathrm{S}}\right) .
$$

Accordingly, the second line is of NNLO order, and we can conclude that formula (4.1) is equivalent to the KrkNLO formula up to NNLO corrections. For a shower where $R_{\mathrm{S}} / R$ is always larger than some value $r$, taking $c \geq 1 / r$ causes eq. (4.1) to reduce to the KrkNLO approach over all of phase space.

The implementation of formula (4.1) goes as follows. The soft events are oversampled by a factor of $c$, and accepted with a probability proportional to the expression in the curly bracket, while the hard events are generated in a standard way.

There are several reasons why it may be of use to have such a family of approaches parameterised by $c$. One is that it is useful to have a parameter to help gauge systematic uncertainties associated with terms that are beyond the accuracy of the method. In fact, the $c$ parameter also gauges the amount of radiation that is multiplied by the inclusive $K$-factor, with respect to the amount that is added in as hard radiation. In this sense, it would play a similar role to the hdamp parameter in POWHEG $[2,26] .{ }^{5}$ Another reason is that the $\theta$-functions in eqs. (3.1), (4.1) can, conceivably, introduce non-smoothness in phase-space coverage even when the underlying $R_{\mathrm{S}}$ and $R$ functions are smooth. Sampling over a range of $c$ values would allow one to address that issue. ${ }^{6}$

So far, the variants that we have discussed involve the rejection of events. For showers with an ordering variable that corresponds to a genuine hardness scale, such as transverse momentum (or any of the scales used in the PanScales showers [8]), it is also possible to envisage a variant where instead of rejecting the event with probability $\frac{R_{\mathrm{S}}-R}{R_{\mathrm{S}}} \theta\left(R_{\mathrm{S}}-R\right)$,

\footnotetext{
${ }^{5}$ Currently other methods are used in the MC@NLO context for the study of matching uncertainties, typically related to the shower starting scale. Whether the use of the $c$ parameter uncertainty can replace these methods is a question that can only be answered in the context of real implementations, and is beyond the scope of the present paper.

${ }^{6} \mathrm{It}$ is not clear to us that this would be a genuine problem. A worse issue is potentially present in the MC@NLO approach (and also in eqs. (3.1) and (4.1)) if a shower has a discontinuity in the distribution of the hardness variable. We are not aware of this having caused significant problems in practice, possibly because subsequent showering smoothens any discontinuities. Were it to be a problem, one solution could be to sample over a range of shower starting scales.
} 
one rejects the shower's first emission with that same probability. If that first emission is rejected, at a value of the ordering variable that we label $t_{1}$, the shower then continues from that scale $t_{1}$, based on an event without the first emission. As the shower continues, each time the shower again attempts to create a new first emission, that emission continues to be rejected with probability $\frac{R_{\mathrm{S}}-R}{R_{\mathrm{S}}} \theta\left(R_{\mathrm{S}}-R\right)$. Once the shower has generated a first emission that is accepted, the shower continues as normal. This ensures that the shower (with first emission rejection) remains unitary. ${ }^{7}$ The Born normalisation factor that multiplies the shower generation then needs to be modified to read

$$
\tilde{B}_{\mathrm{S}}\left(\Phi_{\mathrm{B}}\right)=B_{0}\left(\Phi_{\mathrm{B}}\right)+V\left(\Phi_{\mathrm{B}}\right)+\int \min \left[R(\Phi), R_{\mathrm{S}}(\Phi)\right] \mathrm{d} \Phi_{\mathrm{rad}}
$$

With this variant, the distribution of the hardest radiation is given by

$$
\mathrm{d} \sigma=\tilde{B}_{\mathrm{S}}\left(\Phi_{\mathrm{B}}\right) \tilde{S}\left(t_{\Phi}, \Phi_{\mathrm{B}}\right) \times \frac{\min \left(R(\Phi), R_{\mathrm{S}}(\Phi)\right)}{B_{0}\left(\Phi_{\mathrm{B}}\right)} \mathrm{d} \Phi+\theta\left(R-R_{\mathrm{S}}\right)\left[R-R_{\mathrm{S}}\right] \mathrm{d} \Phi,
$$

where $\tilde{S}\left(t_{\Phi}, \Phi_{\mathrm{B}}\right)$ is the Sudakov form factor that is effectively obtained as a result of the emission rejection procedure,

$$
\tilde{S}\left(t, \Phi_{\mathrm{B}}\right)=\exp \left[-\int_{t_{\Phi}>t} \frac{\min \left[R(\Phi), R_{\mathrm{S}}(\Phi)\right]}{B_{0}\left(\Phi_{\mathrm{B}}\right)} \mathrm{d} \Phi_{\mathrm{rad}}\right] .
$$

As mentioned above, all the expressions in sections 3 and 4 leave out the no-radiation term. For eqs. (3.1) and (4.1), it is easy to verify that the no-radiation term is given by

$$
\mathrm{d} \sigma_{\text {no-rad }}=\bar{B}_{\mathrm{S}}\left(\Phi_{\mathrm{B}}\right) S\left(t_{\text {cut }}, \phi_{B}\right) .
$$

Specifically for eq. (4.1), this comes about by oversampling the events by a factor $c$ and then rejecting no-radiation events with a factor $1 / c$. In the case of eq. (4.2), the no-radiation term is given by

$$
\mathrm{d} \sigma_{\text {no-rad }}=\tilde{B}_{\mathrm{S}}\left(\Phi_{\mathrm{B}}\right) \tilde{S}\left(t_{\text {cut }}, \phi_{B}\right) .
$$

\section{Conclusion}

In this article we have proposed an algorithm for NLOPS generators that, like the MC@NLO approach, relies on the parton shower code for driving the showering steps, but avoids the negative-weight events that complicate the practical use of the MC@NLO approach. The approach can be viewed as a hybrid version of the MC@NLO and KrkNLO methods. The method should be straightforward to implement, requiring no more information than is already used for MC@NLO codes, though it should be noted that it will probably require a closer integration of the showering and fixed-order codes. Following the tradition of associating an acronym to NLOPS methods, a suitable one for this method could be MAcNLOPS, reflecting the combination of Multiplicative and Accumulative (additive) steps.

\footnotetext{
${ }^{7}$ As a matching algorithm to ensure the correct matrix element for the first emission, it bears strong similarities to the algorithm of refs. [27, 28]. That algorithm works within the assumption that one can find some constant $c$ such $c R_{\mathrm{S}}>R$ over the full phase space, evading the need for an additive term.
} 


\section{Acknowledgments}

P.N. wishes to thank Stanislaw Jadach, Andrzej Siódmok, Silvia Ferrario Ravasio, James Whitehead and Giulia Zanderighi for helpful conversations. G.P.S. wishes to thank his collaborators on the PanScales project (Melissa van Beekveld, Mrinal Dasgupta, Frédéric Dreyer, Basem El-Menoufi, Silvia Ferrario Ravasio, Keith Hamilton, Alexander Karlberg, Rok Medves, Pier Monni, Ludovic Scyboz, Alba Soto Ontoso, Grégory Soyez and Rob Verheyen) for discussions on these and related topics. P.N. acknowledges support from Fondazione Cariplo and Regione Lombardia, grant 2017-2070, and from INFN. G.P.S. acknowledges support from a Royal Society Research Professorship (RP $\backslash R 1 \backslash 180112$ ), the European Research Council (ERC) under the European Union's Horizon 2020 research and innovation programme (grant agreement No. 788223, PanScales) and the Science and Technology Facilities Council (STFC) under grant ST/T000864/1.

Open Access. This article is distributed under the terms of the Creative Commons Attribution License (CC-BY 4.0), which permits any use, distribution and reproduction in any medium, provided the original author(s) and source are credited.

\section{References}

[1] S. Frixione and B.R. Webber, Matching NLO QCD computations and parton shower simulations, JHEP 06 (2002) 029 [hep-ph/0204244] [INSPIRE].

[2] P. Nason, A New method for combining NLO QCD with shower Monte Carlo algorithms, JHEP 11 (2004) 040 [hep-ph/0409146] [INSPIRE].

[3] S. Plätzer, Controlling inclusive cross sections in parton shower + matrix element merging, JHEP 08 (2013) 114 [arXiv:1211.5467] [INSPIRE].

[4] L. Lönnblad and S. Prestel, Merging Multi-leg NLO Matrix Elements with Parton Showers, JHEP 03 (2013) 166 [arXiv:1211.7278] [InSPIRE].

[5] S. Jadach, W. Płaczek, S. Sapeta, A. Siódmok and M. Skrzypek, Matching NLO QCD with parton shower in Monte Carlo scheme - the KrkNLO method, JHEP 10 (2015) 052 [arXiv: 1503.06849] [INSPIRE].

[6] E. Boos et al., Generic User Process Interface for Event Generators, in 2nd Les Houches Workshop on Physics at TeV Colliders, Les Houches, France, May 21-June 1, 2001 [hep-ph/0109068] [INSPIRE].

[7] M. Dasgupta, F.A. Dreyer, K. Hamilton, P.F. Monni and G.P. Salam, Logarithmic accuracy of parton showers: a fixed-order study, JHEP 09 (2018) 033 [Erratum ibid. 03 (2020) 083] [arXiv: 1805.09327] [INSPIRE].

[8] M. Dasgupta, F.A. Dreyer, K. Hamilton, P.F. Monni, G.P. Salam and G. Soyez, Parton showers beyond leading logarithmic accuracy, Phys. Rev. Lett. 125 (2020) 052002 [arXiv: 2002.11114] [INSPIRE].

[9] K. Hamilton, R. Medves, G.P. Salam, L. Scyboz and G. Soyez, Colour and logarithmic accuracy in final-state parton showers, JHEP 03 (2021) 041 [arXiv:2011.10054] [INSPIRE].

[10] A. Karlberg, G.P. Salam, L. Scyboz and R. Verheyen, Spin correlations in final-state parton showers and jet observables, Eur. Phys. J. C 81 (2021) 681 [arXiv:2103.16526] [InSPIRE]. 
[11] J.R. Forshaw, J. Holguin and S. Plätzer, Parton branching at amplitude level, JHEP 08 (2019) 145 [arXiv: 1905. 08686] [INSPIRE].

[12] J.R. Forshaw, J. Holguin and S. Plätzer, Building a consistent parton shower, JHEP 09 (2020) 014 [arXiv: 2003.06400] [inSPIRE].

[13] J. Holguin, J.R. Forshaw and S. Plätzer, Improvements on dipole shower colour, Eur. Phys. J. C 81 (2021) 364 [arXiv:2011.15087] [InSPIRE].

[14] Z. Nagy and D.E. Soper, Summations of large logarithms by parton showers, Phys. Rev. D 104 (2021) 054049 [arXiv : 2011.04773] [inSPIRE].

[15] Z. Nagy and D.E. Soper, Summations by parton showers of large logarithms in electron-positron annihilation, arXiv:2011.04777 [INSPIRE].

[16] R. Frederix, S. Frixione, S. Prestel and P. Torrielli, On the reduction of negative weights in MC@NLO-type matching procedures, JHEP 07 (2020) 238 [arXiv:2002.12716] [INSPIRE].

[17] J.R. Andersen and A. Maier, Unbiased Elimination of Negative Weights in Monte Carlo Samples, arXiv:2109.07851 [INSPIRE].

[18] K. Danziger, S. Höche and F. Siegert, Reducing negative weights in Monte Carlo event generation with Sherpa, arXiv:2110.15211 [INSPIRE].

[19] P. Nason and B. Webber, Next-to-Leading-Order Event Generators, Ann. Rev. Nucl. Part. Sci. 62 (2012) 187 [arXiv: 1202.1251] [INSPIRE].

[20] S. Jadach, G. Nail, W. Płaczek, S. Sapeta, A. Siódmok and M. Skrzypek, KrkNLO in HERWIG 7, Acta Phys. Polon. B 48 (2017) 1121 [inSPIRE].

[21] S. Jadach, G. Nail, W. Płaczek, S. Sapeta, A. Siodmok and M. Skrzypek, Monte Carlo simulations of Higgs-boson production at the LHC with the KrkNLO method, Eur. Phys. J. C 77 (2017) 164 [arXiv: 1607. 06799] [INSPIRE].

[22] S. Jadach, W. Płaczek, S. Sapeta, A.K. Siodmok and M. Skrzypek, New simpler methods of matching NLO corrections with parton shower Monte Carlo, PoS LL2016 (2016) 020 [arXiv: 1607.00919] [INSPIRE].

[23] S. Jadach, W. Płaczek, S. Sapeta, A. Siodmok and M. Skrzypek, Parton distribution functions in Monte Carlo factorisation scheme, Eur. Phys. J. C 76 (2016) 649 [arXiv:1606.00355] [INSPIRE].

[24] S. Jadach, W. Płaczek, S. Sapeta, A. Siódmok and M. Skrzypek, NLO Corrections to Hard Process in Parton Shower MC - KrkNLO Method, Acta Phys. Polon. B 46 (2015) 2089 [INSPIRE].

[25] S. Jadach, On the universality of the KRK factorization scheme, Acta Phys. Polon. B 51 (2020) 1363 [arXiv: 2004.04239] [INSPIRE].

[26] S. Alioli, P. Nason, C. Oleari and E. Re, NLO Higgs boson production via gluon fusion matched with shower in POWHEG, JHEP 04 (2009) 002 [arXiv: 0812.0578] [INSPIRE].

[27] M. Bengtsson and T. Sjöstrand, A Comparative Study of Coherent and Noncoherent Parton Shower Evolution, Nucl. Phys. B 289 (1987) 810 [INSPIRE].

[28] M.H. Seymour, Matrix element corrections to parton shower algorithms, Comput. Phys. Commun. 90 (1995) 95 [hep-ph/9410414] [INSPIRE]. 